Spin Physics (SPIN2014)

International Journal of Modern Physics: Conference Series

Vol. 40 (2016) 1660006 (10 pages)

(C) The Author(s)

DOI: $10.1142 /$ S2010194516600065

\title{
Latest Results from the COMPASS Experiment
}

\author{
M. Stolarski \\ on behalf of the COMPASS Collaboration \\ LIP-Lisboa, Avenida Elias Garcia 14 \\ Lisboa, 1000-149, Portugal \\ mstolars@cern.ch
}

Published 29 February 2016

\begin{abstract}
In this paper the latest results from the COMPASS experiment are presented. We show results from longitudinally and transversely polarised targets off which high energy muons are scattered. In addition the future plans of COMPASS as well as results of the beam test runs are also presented.
\end{abstract}

PACS numbers: 13.60.-r, 13.85.-t, 13.88.+e

\section{Introduction}

COMPASS is a fixed target experiment at CERN SPS accelerator. It has an unique possibility to use both muons and hadron beams of both charges. In this paper a brief summary of the COMPASS spin program is presented. In phase-I of the COMPASS experiment naturally polarized positive muons were impinging on a polarized ${ }^{6} \mathrm{LiD}$ or $\mathrm{NH}_{3}$ target. Results on longitudinal and transverse spin asymmetries from this program are presented in the two next sections. In phase-II, which spin program has just started in the fall of 2014, COMPASS will measure the transverse momentum dependent parton distribution functions in the nucleon as well as its generalized parton distribution functions. Some results from beam tests are presented in section 4. Finally, COMPASS has a rich physics program with hadron beams and unpolarized targets, including: pion polarizability from a Primakoff reaction measurement, exotic particles searches and hadron spectroscopy, to name only a few. These results are not covered here.

\section{Longitudinal Physics}

In this section recent results on double spin asymmetry $A_{1}^{p}$ and the polarized structure function $g_{1}^{p}$ are presented. Results of the new COMPASS world $g_{1}$ data NLO

This is an Open Access article published by World Scientific Publishing Company. It is distributed under the terms of the Creative Commons Attribution 3.0 (CC-BY) License. Further distribution of this work is permitted, provided the original work is properly cited. 
QCD fit are also shown. One of the conclusions of this fit is that the gluon polarisation in the nucleon cannot be well constrained even if all the existing inclusive data are used. Therefore, dedicated measurements of the gluon polarization in the nucleon are needed, and such COMPASS results are also presented here.

\subsection{Quark polarization}

From 2002 to 2010 COMPASS used $160 \mathrm{GeV} / \mathrm{c}$ muon beam. For the 2011 run the beam energy was increased to $200 \mathrm{GeV} / \mathrm{c}$ to access lower values of the Bjorken scaling variable, $x$, while still in the perturbative region, i.e. keeping the negative four momentum transfer $Q^{2}$, above $1(\mathrm{GeV} / \mathrm{c})^{2}$. The obtained spin asymmetry $A_{1}^{p}$ is presented in the left panel of Fig. 1. Asymmetry is compatible with results from the 2007 run with lower beam energy. Studies of semi-inclusive asymmetries for $h^{ \pm}$, and identified $\pi^{ \pm}, K^{ \pm}$are ongoing.

It is worth adding that COMPASS also studied the low $x$ behaviour of $A_{1}^{p}$ for $Q^{2}<1(\mathrm{GeV} / \mathrm{c})^{2}$. Compared to previous experiments the statistical uncertainties were reduced ten-fold, while a wider domain in $x$ is studied. Even at low values of $x$ asymmetry is found to be positive, around $1 \%$, see the right panel of Fig. 1 . The same measurement performed in COMPASS on the deuteron target gave results consistent with zero. ${ }^{1}$

From inclusive asymmetries the $g_{1}^{p}$ structure functions were calculated. These new results together with the world $g_{1}^{p, d, n}$ measurements were used in the new COMPASS NLO fit. The results of fitted singlet $\Delta q_{S}, \Delta g$ and other quark combinations are presented in Fig. 2. Due to the large correlation between $\Delta g$ and $\Delta \Sigma$ the current world data on inclusive $g_{1}$ structure function cannot disentangle between positive and negative gluon polarisation in the nucleon, and related $\Delta \Sigma$ behaviour at low $x$. Dedicated measurements of $\Delta g / g$ in a semi-inclusive analysis are presented in the next sub-section. The COMPASS $g_{1}^{p}$ and $g_{1}^{d}$ were also used to test the so called Bjorken Sum Rule. This sum relates the first moment of the non-singlet part of $g_{1}$ with a ratio of axial and vector couplings, $g_{A} / g_{V}$. The COMPASS result,
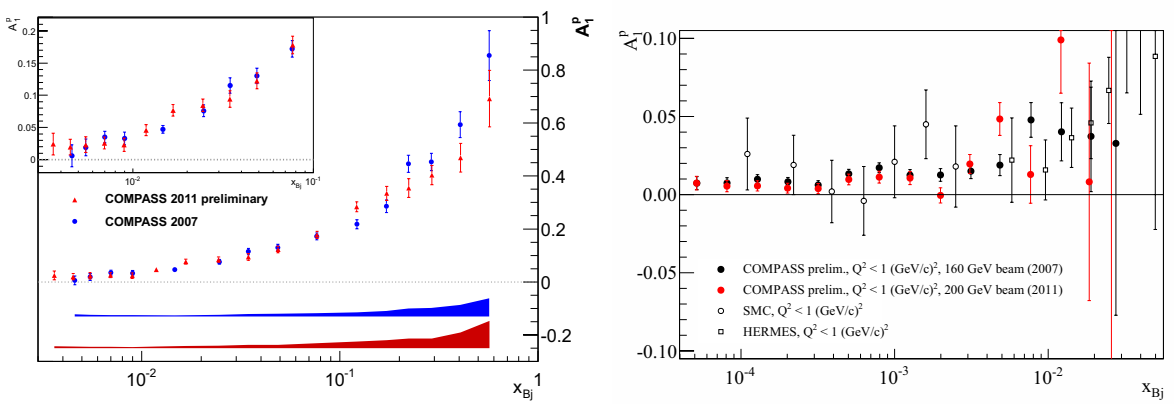

Fig. 1. Spin dependent asymmetry $A_{1}^{p}$, left panel: $Q^{2}>1(\mathrm{GeV} / \mathrm{c})^{2}$; right panel: $Q^{2}<1$ $(\mathrm{GeV} / \mathrm{c})^{2}$ 

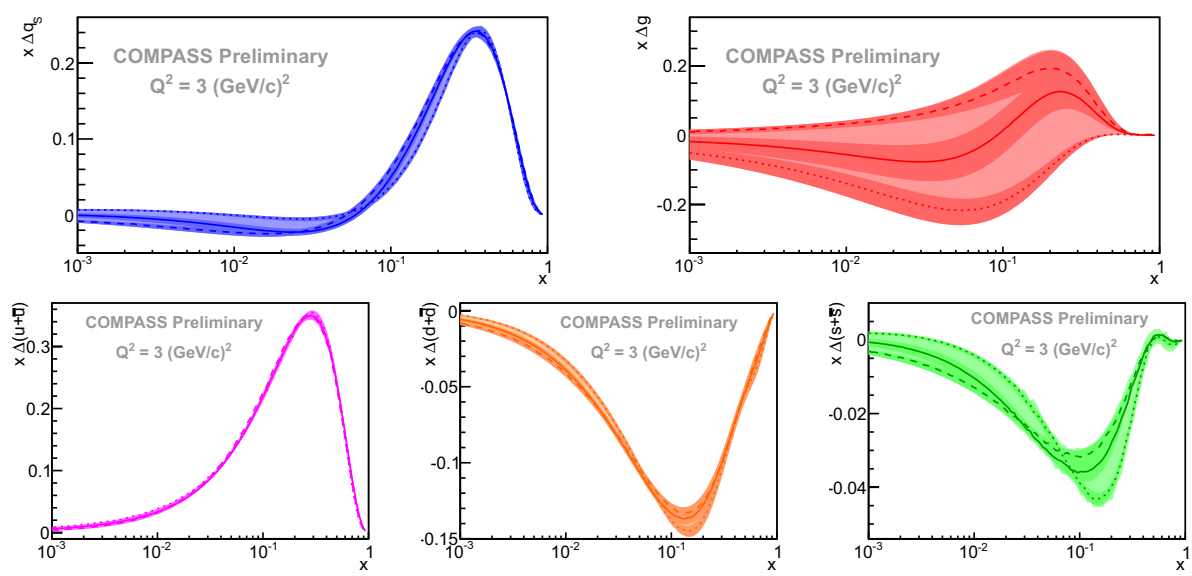

Fig. 2. Results of COMPASS NLO QCD fit to world $g_{1}$ data.

$g_{A} / g_{V}=1.219 \pm 0.052 \pm 0.095$ is in excellent agreement with more precise results from neutron $\beta$ decay, ${ }^{2}$ which gives $g_{A} / g_{V}=1.2701 \pm 0.0025$.

It is worth adding that a low value of the first moment of $\Delta \Sigma$, assuming $\mathrm{SU}(3)$ symmetry, leads to large negative polarisation of the strange quarks in the nucleon. The strange polarisation can also be studied in semi-inclusive events with kaon observed in the final state. The strange quark polarization obtained from SIDIS data $^{3,4}$ is hardly compatible with the negative expectation from the DIS analysis.

However, the extraction of $\Delta s(x)$ in a SIDIS analysis strongly depends upon the assumed fragmentation functions $(\mathrm{FF})$. In the analyses mentioned before the DSS FF set was used. ${ }^{5}$ In case the ratio of fragmentation functions $D_{\bar{s}}^{K^{+}} / D_{u}^{K^{+}}$is smaller than in DSS, consistent results for inclusive and semi-inclusive analyses. One possibility to study aforementioned FF is to measure kaon multiplicities as a function of $x$. The sum of charged kaons multiplicities is especially well suited to study $D_{\bar{s}}^{K^{+}}$. In LO the observed multiplicity is proportional to $\int D_{Q}^{K}(z) d z+$ $S / Q \int D_{S}^{K}(z) d z$, where $Q=u+\bar{u}+d+\bar{d}, S=s+\bar{s}$. Therefore at high $x$, where S/Q is low, one has an access to $\int D_{Q}^{K}(z) d z$ while at lower $x$, the impact of $S / Q \int D_{S}^{K}(z) d z$ should be clearly visible if $D_{\bar{s}}^{K^{+}} / D_{u}^{K^{+}}$is large. The COMPASS preliminary results are presented in Fig. 3. The expected rise of the multiplicity sum at low $x$ is not seen. COMPASS data seem to prefer lower values of $D_{\bar{s}}^{K^{+}} / D_{u}^{K^{+}}$than expected from DSS. Finally, COMPASS preliminary results do not agree neither in shape nor in magnitude with HERMES final results. ${ }^{6}$ For more details concerning this sub-section refer to two contributions by F. Kunne in these proceedings.

\subsection{Gluon polarisation}

COMPASS has performed several direct gluon extraction measurements. ${ }^{7-9}$ Since the leading order photo-absorption process is not sensitive to $\Delta g / g$ one has to study 


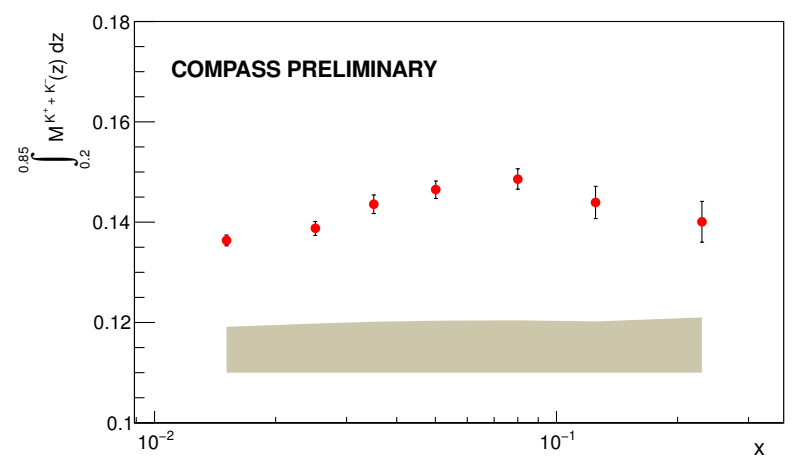

Fig. 3. Kaon multiplicity sum as a function of $x$, see text for details.

higher order processes like photon-gluon fusion (PGF) to access $\Delta g$. For example in LO a clean sample of PGF are events with charmed mesons produced in the final state. The data sample containing high- $p_{T}$ momentum hadrons is also enriched in PGF events.

Recently, the data used in, ${ }^{8}$ were reanalyzed using a new method $\left(\right.$ all- $p_{T}$ method). This method allows for the simultaneous extraction of $\Delta g / g$ and the leading process asymmetry $A_{1}$ from the same data set. As a result the systematic and statistical errors are reduced comparing to the situation when the values of $A_{1}$ asymmetry were taken from as from external source as in. ${ }^{8}$

The preliminary results on $\Delta g / g$ obtained with all- $p_{T}$ method are presented in the left panel of Fig. 4 in three intervals of gluon momentum fraction $x_{g}$. These results are compared with world $\Delta g / g$ obtained in LO analyses. COMPASS data suggest that the $\Delta g / g$ is positive in the measured range of $x_{g}$ with an average
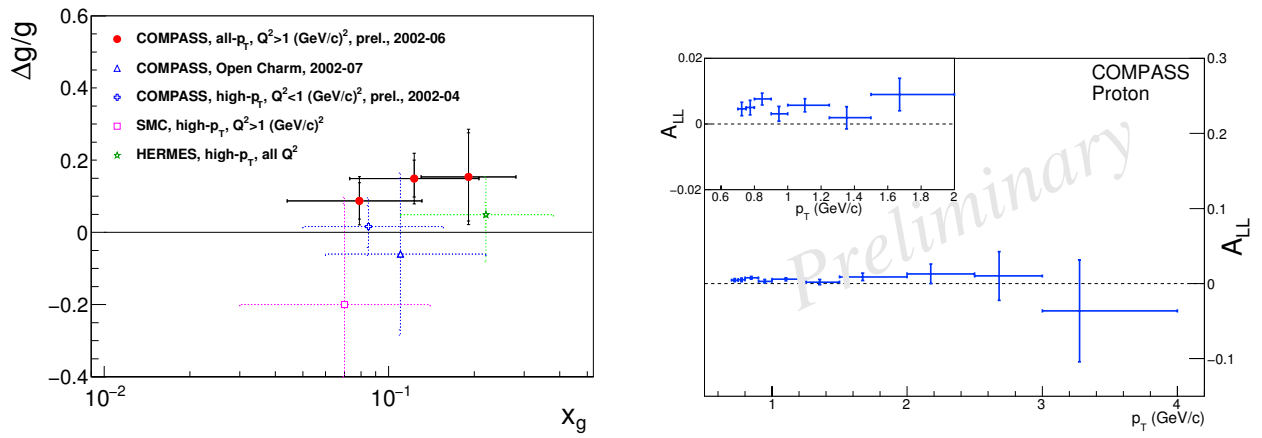

Fig. 4. Left panel: Comparison of the extracted $\Delta g / g$ from the so called all- $p_{T}$ method with world LO analyses; Right panel: the values of the single inclusive spin dependent asymmetry $A_{L L}$ as a function of hadron $p_{T}$. 
$\Delta g / g=0.113 \pm 0.038 \pm 0.035$ at average scale $\mu^{2}=3(\mathrm{GeV} / \mathrm{c})^{2}$ and average $x_{g}=0.10$.

To further constrain $\Delta g / g$ the single inclusive hadron asymmetries were extracted from a low $Q^{2}$ data sample, where the hard scale is assured by large transverse momentum of the produced hadron. As an example the extracted asymmetries for the proton target are presented in the right panel of Fig. 4. The obtained results can also be compared with NLO collinear approach calculations. ${ }^{10}$ Preliminary COMPASS results are compatible with zero for (not shown here) deuteron target and slightly positive for the proton target. However, in the high $p_{T}$ region, for proton target theory expects higher asymmetries even in the scenario with maximum positive gluon polarisation. This tension between data and theory is being investigated. For more details concerning COMPASS $\Delta g / g$ measurements see the contribution by K. Kurek in these proceedings.

\section{Transverse Physics}

Studies of transversely polarised nucleons give access to transverse momentum dependent structure functions which are crucial to understand the $3-\mathrm{D}$ picture of the nucleon.

A known Sivers effect, which is related with parton intrinsic transverse momentum, was recently measured in COMPASS for identified hadrons $\pi^{ \pm}, K^{ \pm, 0}$. In the valence region about $3 \%$ effect is observed for $\pi^{+}$and somewhat larger for $K^{+}$. Similarly Collins effect, related to hadron fragmentation $p_{T}$, is found to be non-zero for both $\pi^{+}$and $\pi^{-}$. For the kaon case more data is needed to give a decisive answer. The COMPASS results for both Sivers and Collins effects are presented in left and right panels, respectively, of Fig. 5 as functions of $x, p_{T}$ and $z .^{11}$
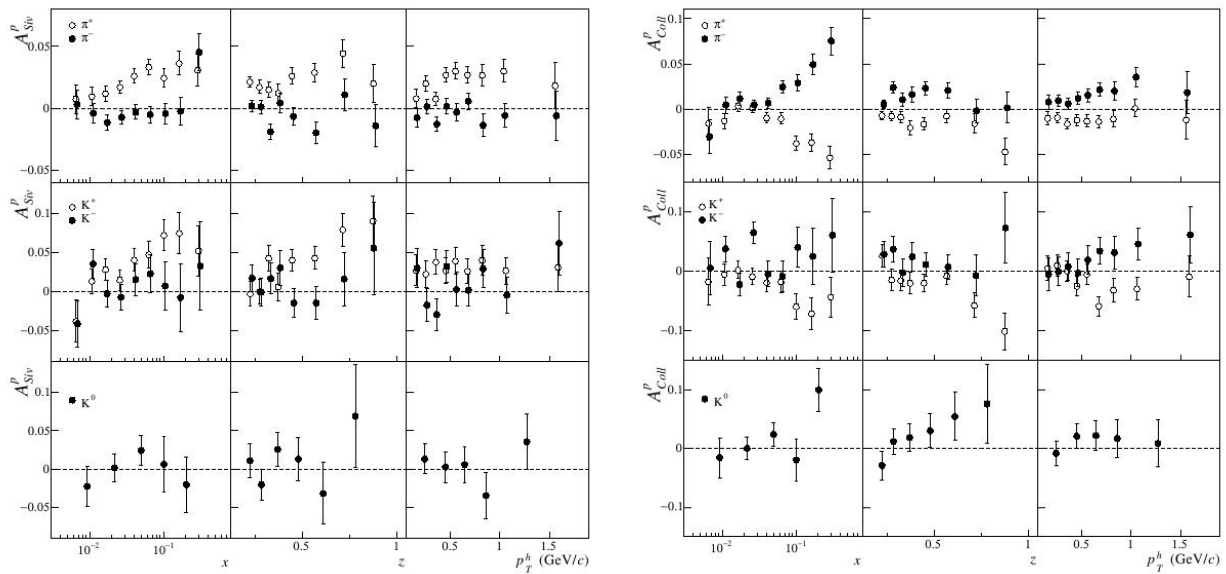

Fig. 5. Extracted Sivers and Collins asymmetries for identified hadrons, left and right panels respectively. 
The Sivers effect measurement were so far mostly related with quarks. In a similar way as in the measurements of $\Delta g / g$, by selecting a sample with high $p_{T}$ hadrons observed in the final state one can access the gluon Sivers function. Such a measurement was recently performed in COMPASS. The preliminary result based on part of the COMPASS statistics is compatible with zero, $A_{P G F}^{\sin \left(\phi_{2 h}-\phi_{S}\right)}=-0.14 \pm$ $0.15 \pm 0.06$. Results containing all COMPASS statistics are expected soon. For more details see the contribution by A. Szabelski and K. Kurek in these proceedings.

Interesting features are found in studies of two hadron $(2 h)$ asymmetries, which give access to the interference FF. They were recently measured for identified pairs of $\pi^{+} \pi^{-}, K^{+} K^{-}, \pi^{+} K^{-}$and $K^{+} \pi^{-}$. They are presented in Fig. 6 as a function of $x, p_{T}$ and invariant mass of the hadron system $\left(M_{i n v}\right)$. The knowledge of $2 h$ asymmetries for proton and deuteron targets allows the extraction of the transversity $h_{1}$. The obtained preliminary results are presented in the left panel of Fig. 7 and are compared to a model ${ }^{12}$ which uses only single hadron Collins asymmetry. A fair agreement between the extracted transversity from $2 h$ analysis and the model is seen. For more details see the contribution by G. Sbrizzai in these proceedings.

Another very interesting study is a comparison of $2 \mathrm{~h}$ asymmetries with $1 \mathrm{~h}$ Collins asymmetry, which is presented in the right panel of figure 7. A very striking similarity is observed between the two asymmetries. An important theory achievement is the newly derived cross section formula for the $l^{ \pm} p \rightarrow l^{ \pm}+2 h+X$ process, that allows study of asymmetries as a function of the azimuthal angle difference between the two hadrons. Within this formalism one can predict $2 \mathrm{~h}$ asymmetries from the knowledge of $1 \mathrm{~h}$ Collins asymmetry. The expected ratio of the two asymmetries

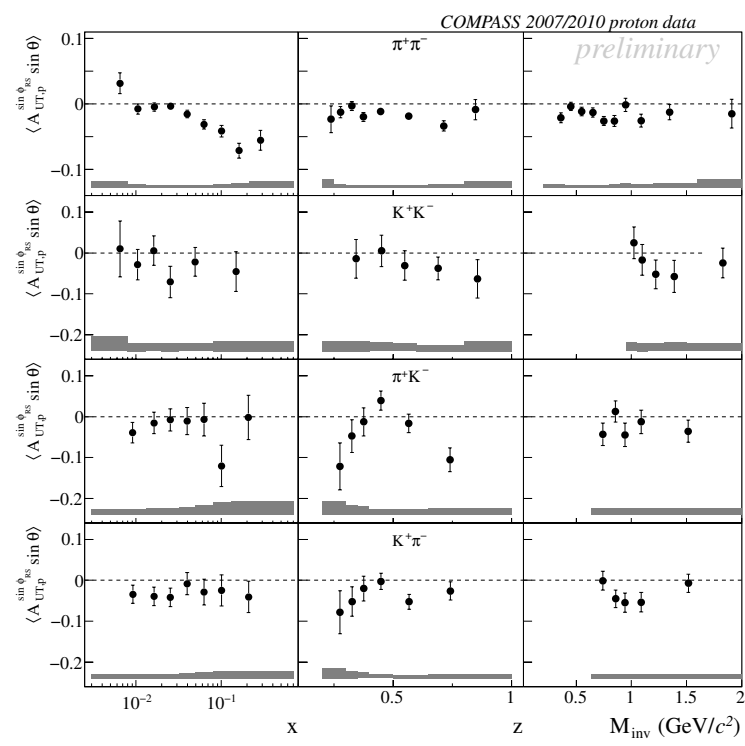

Fig. 6. Two hadron asymmetry results for pairs of identified hadrons of opposite charge. 

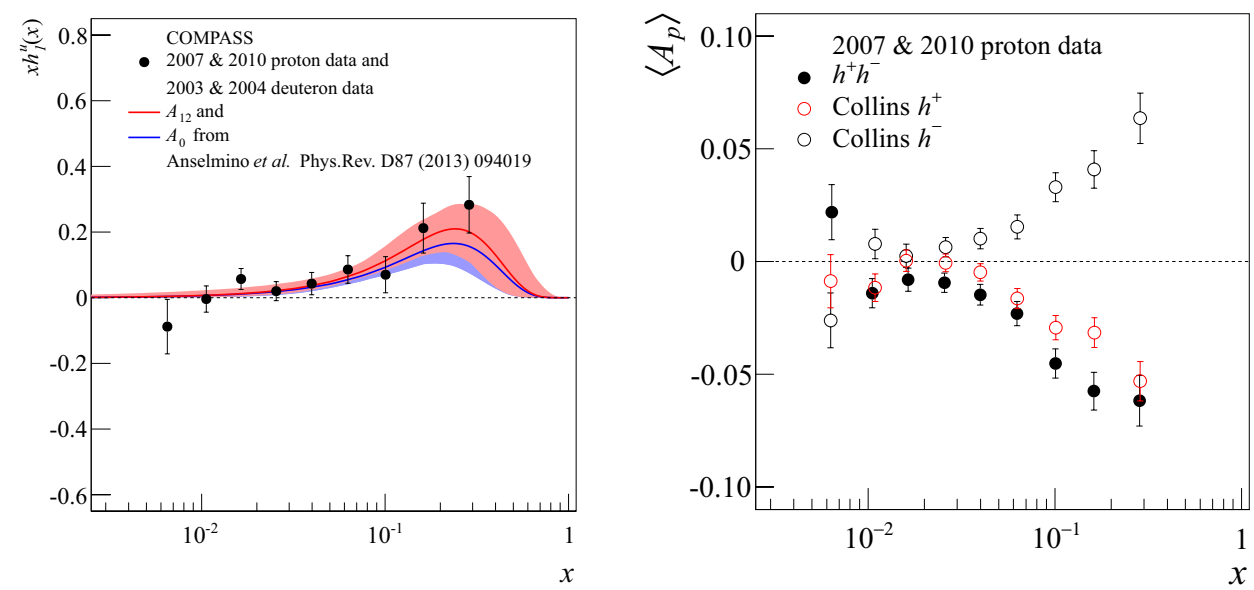

Fig. 7. Left panel: An example of extracted values of transversity $h_{1}^{u_{v}}(x)$ and comparison with a model; Right panel: Comparison of $2 h$ asymmetries with $1 h$ Collins asymmetry.

at high $x$ is about $4 / \pi$, in agreement with the experimental measurement. See the contribution by F. Bradamente in these proceedings for more details.

A rapid development of transverse momentum dependent domain asks for multidimensional analyses. These were started in COMPASS and the data are being scrutinized. The example of the bidimensional analysis of the Sivers asymmetry in intervals of $Q^{2}$ and $x, z, p_{T}$, or the invariant mass of the hadron system, $W$, is presented in Fig. 8. For more details see the contribution by B. Parsamyan in these proceedings. At the same time COMPASS also analyses hadron multiplicities as functions of $x, Q^{2}, z$ and most importantly $p_{T}$, so that better understanding of intrinsic quark $k_{T}$ and fragmentation $p_{\perp}$ can be achieved. For more details see the contribution by N. Makke in these proceedings.

So far mostly Sivers and Collins effects were discussed. However, COMPASS measured also the six other transverse asymmetries which appear in the LO general SIDIS cross-section formula. All but one were found to be consistent with zero. The non zero one $A_{U T}^{\sin \phi_{S}}$ is related to Sivers $f_{1 \perp}$ and transversity $h_{1}$.

\section{COMPASS Phase-II}

In the fall of 2014 COMPASS restarted data taking after two years of shut-down of CERN accelerators due to LHC upgrade. The main goal of the forthcoming 2015 run are studies of transverse momentum dependent structure functions by means of the Drell-Yan process induced by a negative pion beam on a transversely polarised proton target. One of the important expectations is the predicted change of sign of Sivers and Boer-Mulders functions between SIDIS and Drell-Yan processes.

In order to collect a large amount of clean Drell-Yan events, the COMPASS spectrometer had to be modified. A hadron absorber was added directly downstream 


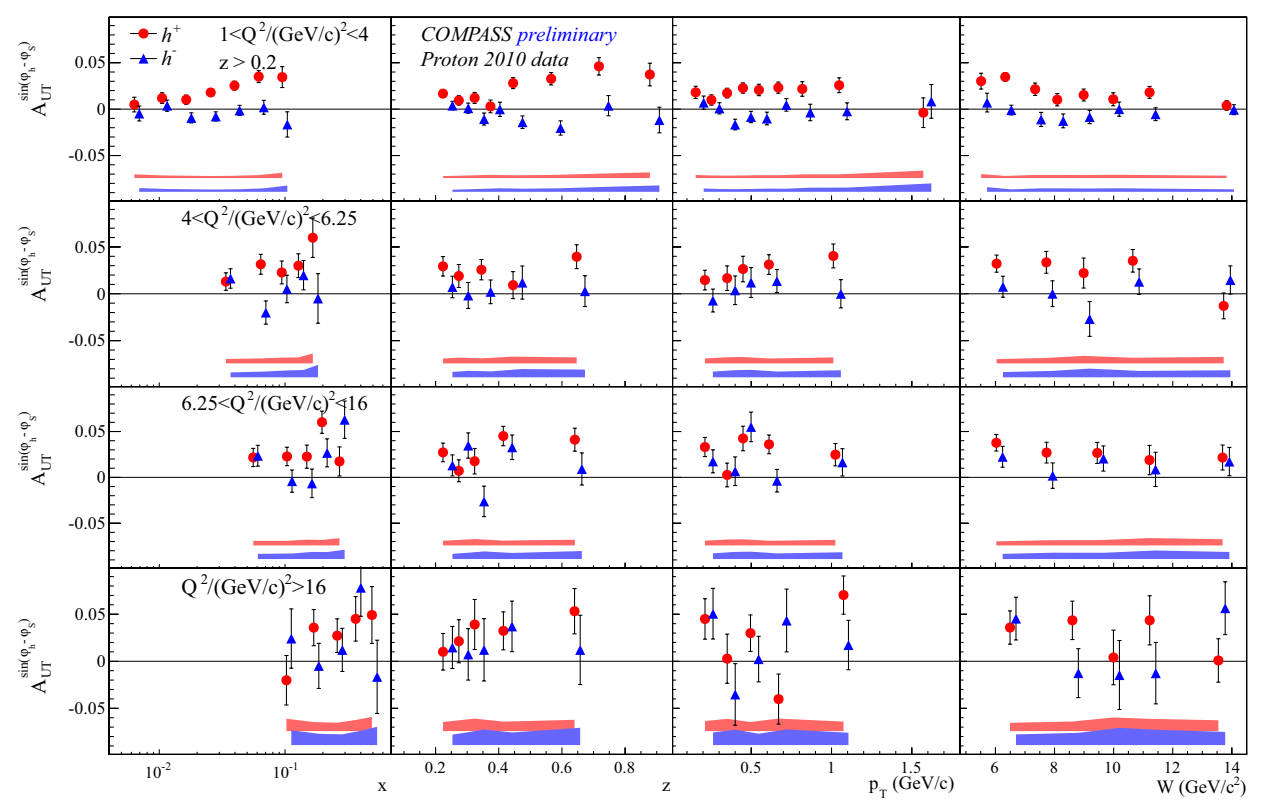

Fig. 8. Measured Sivers effect in bidimensional grid of $Q^{2}$ and $x, z, p_{T}$, or $W$.

the target. Although, in this way the multiple-scattering increase one has verified that the mass resolution of the dimuon spectrum is not too much degraded. Moreover, to study polarized effects COMPASS simultaneously collects data with target cells polarised in opposite directions. Therefore it is also mandatory that the vertex resolution along the beam line direction is good enough to separate the two cells.

The results of a beam test performed in 2009 are presented in Fig. 9, being satisfactory in what concerns these two problems. compared to a prototype absorber used in 2009 test, The final one is expected to improve both the mass resolution and the vertex resolution. The vertex resolution will be further reduced by installing a dedicated vertex detector in the middle of the hadron absorber.

COMPASS aims to collect totally 180000 events of Drell-Yan in the high dimuon mass region 4-9 GeV/ $\mathrm{c}^{2}$. The expected statistical errors for various asymmetries are presented in left panel of Fig. 10. An example of the theoretical prediction ${ }^{13}$ is shown in the right panel of Fig. 10. If the asymmetries are as large as predicted by some theory groups, COMPASS should observe rather significant non zero results. For more details see the contribution by B. Parsamyan in this proceedings. Details about possible unpolarized Drell-Yan studies using data to be collected and (additional) $\mathrm{Al}$ and $\mathrm{W}$ targets are presented in paper by W.-C. Chang in these proceedings.

Other COMPASS plans include Generalized Parton Distribution (GPD) measurements, especially in the golden channel of Deeply Virtual Compton Scattering (DVCS). The GPD give also information about the 3-D structure of the nucleon, 

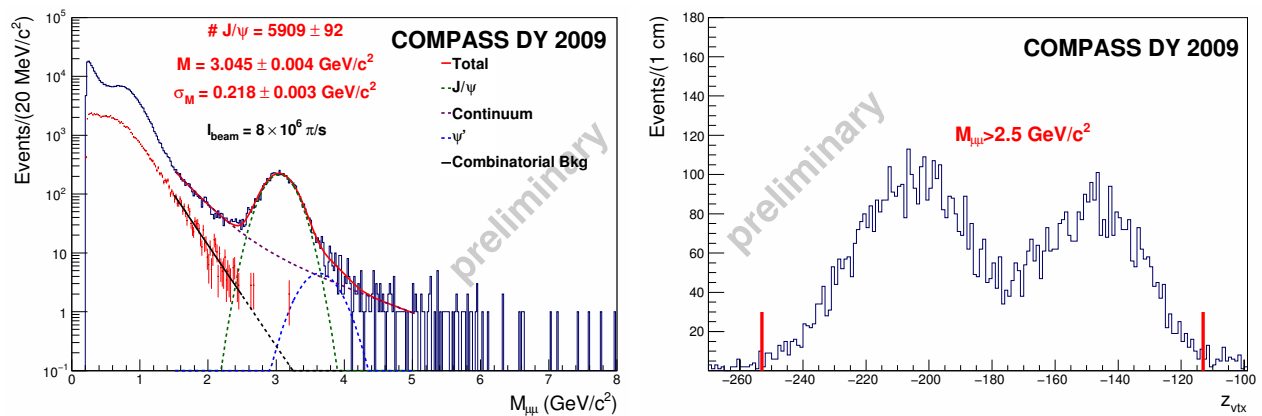

Fig. 9. Left panel: Mass resolution of $J / \Psi$ obtained from a test with hadron absorber, Right panel: separation of interaction vertex between two target cells.
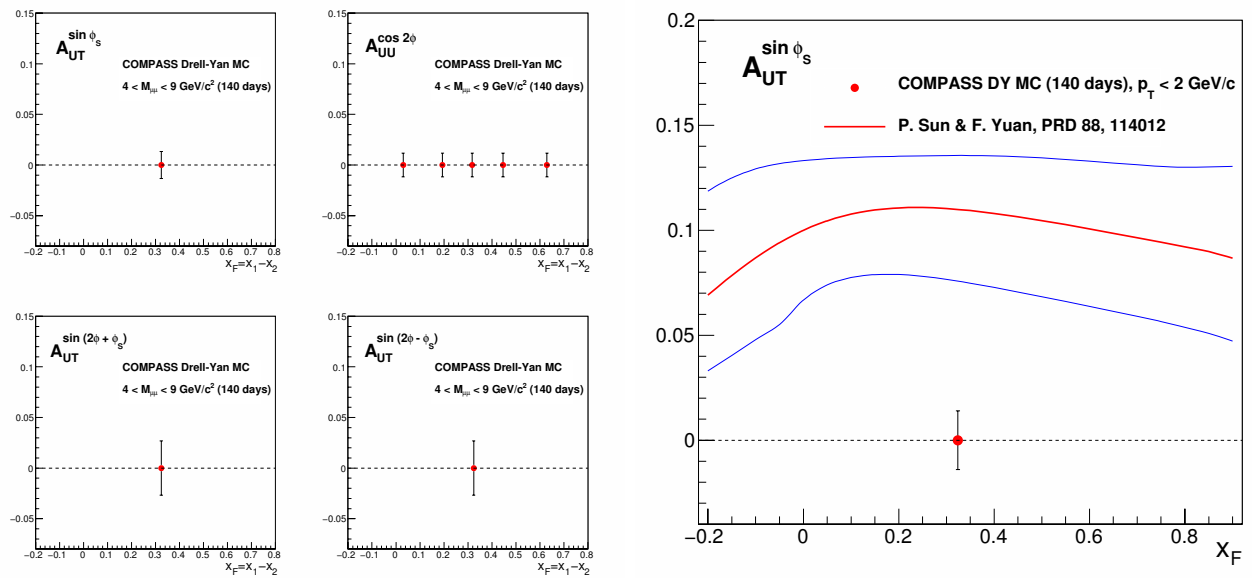

Fig. 10. Left panel: expected statistical errors in each of the relevant azimuthal modulations; Right panel: comparison of the expected precision for the Sivers effect with a model prediction.

with one of the dimension being the impact parameter space. Some of the integrals involving GPD are connected with angular momentum of quarks in the nucleon.

A pilot run data were taken in 2012. The obtained results, seen in Fig. 11, show that indeed an excess of events attributed to DVCS above the Bethe-Heitler $(\mathrm{BH})$ background is observed in high $x$ region, as expected. For more details see the contribution by A. Ferrero in these proceedings.

The generalized parton distributions can be also obtained from studies of hard exclusive vector meson production. As usually various modulations of the cross section are sensitive to different combinations of GPD. Such studies were performed in COMPASS, so far for the $\rho$ meson (studies for $\omega$ meson are being finalized). The extracted asymmetries are generally consistent with zero, except for some deviation observed for $A_{U T}^{\sin _{\phi_{s}}}$. The asymmetries are presented in the right panel of Fig. 11. For more details see the contribution from J. ter Wolbeek in these proceedings. 

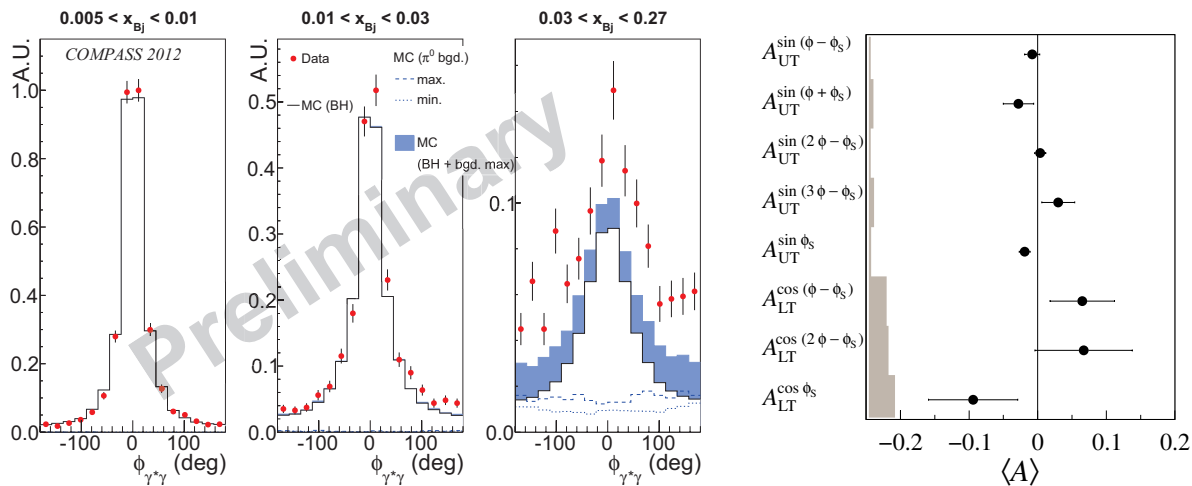

Fig. 11. Left panel: Extracted spectra for events with hard exclusive photon in the final state in three intervals of $x$. Clear indication of DVCS events is visible in the highest $x$ region. Right Panel: Azimuthal asymmetries extracted from hard exclusive $\rho$ production, each of which is related to a different combination of GPD.

\section{Summary}

In this paper a summary of recent results from the COMPASS experiment was presented. COMPASS is finalizing analyses on longitudinally and transversely polarised target; several new important results are expected to be published soon.

After two years of shut-down, last fall COMPASS restarted data taking. The main goal of the 2015 run is to study the transverse momentum dependent parton distribution functions in Drell-Yan process. This will be followed by a 2016-17 data taking concentrated on studies of Generalized Parton Distribution functions, mainly using the deeply virtual Compton scattering process.

\section{Acknowledgments}

This research was supported by the Portuguese Fundação para a Ciência e a Tecnolagia, grant SFRH/BPD/64853/2009.

\section{References}

1. COMPASS Collab. (V.Yu. Alexakhin et al.), Phys. Lett. B 647, 330 (2007).

2. Particle Data Group (J. Beringer et al.), Phys. Rev. D 86, 010001 (2012).

3. HERMES Collab. (A. Airapetian et al.), Phys Lett. B 666, 446 (2008).

4. COMPASS Collab. (M. Alekseev et al.), Phys. Lett. B 680, 217 (2009).

5. D. de Florian, R. Sassot and M. Stratmann, Phys. Rev. D 75, 114010 (2007).

6. HERMES Collab. (A. Airapetian et al.), Phys. Rev. D 87, 074029, (2013).

7. COMPASS Collab. (E. S. Ageev et al.), Phys. Lett. B 633, 25 (2006).

8. COMPASS Collab. (C. Adolph et al.), Phys. Lett. B 718, 922, (2013).

9. COMPASS Collab. (C. Adolph et al.), Phys. Rev. D 87, 052018, (2013).

10. B. Jaeger, M. Stratmann, and W. Vogelsang, Eur. Phys. J. C 44, 533 (2005).

11. COMPASS Collab. (C. Adolph et al.), hep-ex/1408.4405 subm. to Phys. Lett. B

12. M. Anselmino et al., Phys. Rev. D 87, 094019 (2013).

13. P. Sun and F. Yuan, Phys. Rev. D 88, 114012 (2013). 